\title{
Inductive Spikes in the Crab Nebula: A Theory of $\gamma$-Ray Flares
}

\author{
John G. Kirk ${ }^{*}$ and Gwenael Giacinti ${ }^{\dagger}$ \\ Max-Planck-Institut für Kernphysik, Saupfercheckweg 1, 69117 Heidelberg, Germany
}

(Received 29 August 2017; published 21 November 2017)

\begin{abstract}
We show that the mysterious, rapidly variable emission at $\sim 400 \mathrm{MeV}$ observed from the Crab Nebula by the AGILE and Fermi satellites could be the result of a sudden drop in the mass loading of the pulsar wind. The current required to maintain wave activity in the wind is then carried by very few particles of a high Lorentz factor. On impacting the nebula, these particles produce a tightly beamed, high-luminosity burst of hard gamma rays, similar to those observed. This implies that (i) the emission is synchrotron radiation in the toroidal field of the nebula and, therefore, linearly polarized and (ii) this mechanism potentially contributes to the gamma-ray emission from other powerful pulsars, such as the Magellanic Cloud objects J0537-6910 and B0540-69.
\end{abstract}

DOI: 10.1103/PhysRevLett.119.211101

The detection of powerful gamma-ray flares from the Crab Nebula by the AGILE satellite and the Large Area Telescope on the Fermi satellite [1-3] has provided theorists with three major puzzles: How are particles able to emit synchrotron radiation well above the $\sim 100 \mathrm{MeV}$ astrophysical "upper limit" [4]? What is the geometry and location of the source, given that it varies on a time scale of hours, whereas the nebula has a light-crossing time of months? By which mechanism can such a small source achieve a power only one order of magnitude less than that of the entire nebula? Many theories have addressed these issues (for a review, see [5]), but none has yet achieved general acceptance. In this Letter, a novel theory is proposed, based on the properties of relativistic winds that are dominated by Poynting flux: The frequency, variability, and power of the flares emerge as natural consequences of a sharp reduction of the supply of electron-positron pairs to the wind of the Crab pulsar, an effect closely analogous to the voltage spikes generated when the current in an inductive electrical circuit is interrupted. Although the underlying cause remains a mystery, such interruptions are not implausible, since the electromagnetic cascades responsible for creating the pairs are thought to be highly erratic [6], a conclusion supported by strong pulse-to-pulse fluctuations in the radio emission, that presumably originates in this region [7]. The new theory predicts the polarization properties of the flares, which may be measurable in the near future [8], and suggests that a similar emission may be detectable from other pulsar wind nebulae.

Published by the American Physical Society under the terms of the Creative Commons Attribution 4.0 International license. Further distribution of this work must maintain attribution to the author(s) and the published article's title, journal citation, and DOI.
The Crab Nebula is powered by an electron-positron wind that is energetically dominated by electromagnetic fields. These oscillate at the pulsar period $P$ and have a finite phaseaveraged (dc) component [9], the necessary currents being carried by the pairs. Such winds propagate radially [10] up to a termination shock at radius $r=r_{\mathrm{ts}}$, where the ram pressure balances that of the surroundings. Because the particle density drops off as $1 / r^{2}$, nonmagnetohydrodynamics effects become important at a sufficiently large radius. These can lead to the conversion of the oscillations into electromagnetic waves [11,12], for which there is no observational evidence, or to damping of the oscillations, accompanied by radial acceleration of the plasma.

This latter process was originally modeled as magnetic reconnection in a "striped wind"-one with oppositely directed bands of toroidal magnetic field separated by hot current sheets $[13,14]$. Dissipation in the current sheets releases the magnetic tension, leading to radial acceleration of the flow. However, in the case of the Crab, complete dissipation of the wave energy occurs only for a relatively high pair density [15], which implies a terminal Lorentz factor $<10^{4}$. An additional acceleration mechanism is then needed for the flares [16]. Conversely, lower-density flows reach higher bulk Lorentz factors, even though they do not achieve complete dissipation. However, in the Crab, hot plasma cannot be confined in the sheets up to the termination shock [14], which invalidates this model and leaves the ultimate fate of the waves uncertain. Here, we present a solution to this problem: Assuming that the pulsar wind is launched as a mildly supersonic magnetohydrodynamic (MHD) flow with embedded magnetic fluctuations, we demonstrate that inductive acceleration converts $10 \%$ of the power into kinetic energy. When the supply of electronpositron pairs is severely limited, the few particles that are present achieve very high Lorentz factors.

Dissipation is not essential for acceleration [17], as was shown using a static, sinusoidal, magnetic shear (a "sheet 
pinch") as a wave model [18]. This wave contains neither a current sheet nor hot plasma. The transverse magnetic field has a constant magnitude and rotates at a uniform rate as a function of the phase. The current is carried by cold electron and positron fluids which, in the comoving frame, flow along the magnetic field. Provided the transverse flow speed $v_{\perp}$ remains small, the wave propagates at constant speed, as expected in MHD. But towards a larger radius the drop in density causes $v_{\perp}$ to increase to maintain the wave currents. When $v_{\perp} \approx c$, particle inertia causes a small misalignment of the current and field, leading to a net radial acceleration. Because resistive dissipation is absent and the underlying cause is the maintenance of a current flow, this mechanism is appropriately described as inductive acceleration.

Reference [18] identified the location of the inductive acceleration zone and gave an approximate solution in which the Lorentz factor $\gamma$ of the plasma is proportional to $r$. This result is not immediately applicable to a pulsar wind, because it does not allow for a dc component. However, it is straightforward to generalize the model to that of a striped wind that contains two sheet pinches instead of two hot current sheets. Each pinch causes the magnetic field to rotate through $\pi$ radians, so that the field outside of the pinches is purely toroidal, and the magnitude of the dc component is controlled by the location in phase of the pinches. The analysis is particularly simple if the thickness of the pinches is small-i.e., they become rotational discontinuities. This wave displays the same radial evolution as the single, sinusoidal pinch studied by Ref. [18] (for details, see Supplemental Material [19]); the only difference is that the dc component is $\propto 1 / r$, which causes the pinches to migrate in phase. Ultimately, when the wave energy has been completely converted into kinetic energy, the pinches merge. However, provided their initial separation is not small, merging occurs only towards the end of the acceleration phase, when the kinetic energy flux is already comparable to the Poynting flux.

Pulsar winds are usually modeled as either isotropic or axisymmetric, with the power concentrated towards the equator [20], but the estimates presented below are not sensitive to this distinction. Assuming isotropy, two parameters characterize the wind: (i) the ratio at the light cylinder, $r_{L}=c P / 2 \pi$, of the gyrofrequency of a nonrelativistic electron to the rotation frequency of the neutron star, given, for a magnetically dominated flow, by

$$
a_{L}=\left(e^{2} L_{\mathrm{sd}} / m^{2} c^{5}\right)^{1 / 2}=3.4 \times 10^{10} L_{38}^{1 / 2},
$$

where $L_{\text {sd }}=L_{38} \times 10^{38} \mathrm{erg} \mathrm{s}^{-1}$ is the spin-down power of the neutron star, and (ii) the energy carried per particle in units of $m c^{2}$

$$
\mu=L_{\mathrm{sd}} /\left(\dot{N}_{ \pm} m c^{2}\right)
$$

where $\dot{N}_{ \pm}$is the rate at which electrons and positrons are transported into the nebula by the wind. For the Crab, $a_{L} \approx 7.6 \times 10^{10}$, but $\mu$ is uncertain. It can be related to the "multiplicity" parameter $\kappa$ used in modeling pair production near the pulsar [6] [conventionally, one sets $\kappa=$ $a_{L} /(4 \mu)$ [14]], but this should not be interpreted too literally. A latitude-dependent mass loading of the wind can be defined by generalizing (2), which enables the detailed modeling of the radio to $\mathrm{x}$-ray emission of the Crab Nebula [21]. The tightest constraints, however, refer to the average of $\mu$ over the entire wind and over the lifetime of this object: $10^{4} \lesssim \mu \lesssim 10^{6}$, which corresponds to $\dot{N}_{ \pm} \approx$ $10^{39}-10^{41} \mathrm{~s}^{-1}$ and $10^{6} \gtrsim \kappa \gtrsim 10^{4}$ [22].

The radial evolution of the wind is governed by three equations derived in Ref. [18] and in Supplemental Material [19] [where they are numbered (35), (37), and (38)]. These can be integrated numerically to find the three unknown functions of $r$, which are the fluid Lorentz factor $\gamma$, the "magnetization parameter" $\sigma=|B|^{2} /\left(8 \pi n_{0} \gamma^{2} m c^{2}\right)$, with $n_{0}$ the proper number density of the fluids and $B$ the magnetic field strength, and the transverse component of the dimensionless fluid four-velocity $u_{\perp}=v_{\perp} /\left(c^{2}-v_{\perp}^{2}\right)^{1 / 2}$. Despite impressive progress based on 3D MHD and force-free simulations [23], the nature of the wind at launch and, therefore, the initial conditions remain uncertain. Fundamental properties of axisymmetric flows suggest they accelerate steadily up to the sonic point and thereafter coast at a constant speed [10,24,25] (see Supplemental Material [19]). Assuming the wave is launched as a mildly supersonic MHD flow, Fig. 1 shows results for $a_{L}=7.6 \times 10^{10}$ and two different values of mass loading: $\mu=10^{6}$, the time-averaged value needed to provide the optical to x-ray emitting particles in the Crab Nebula, and $\mu=a_{L}$, a severely charge-depleted value with $\kappa \approx 1 / 4$. The latter is a plausible upper limit on $\mu$, since it corresponds to the charge density at which lightlike waves can propagate already at radius $r_{L}$. If these predominate, the solutions shown in Fig. 1 lose validity. However, embedded fluctuations could, in principle, be preserved at even lower density - a more rigorous but higher upper limit, $\mu \leq a_{L}^{3 / 2}$, is derived in Eq. (40) of Supplemental Material [19]. Note that a fluctuation in pair loading need not be isotropic and is advected radially with the flow. Sectors of the wind separated by an angle $>1 / \gamma$ evolve essentially independently of each other, since a light signal launched by a fluid element in one sector does not reach the equivalent element in the other until it has more than doubled its radius.

The results shown in Fig. 1 exhibit three phases. In order of increasing radius, these are (i) the MHD phase, in which $u_{\perp}$ is small and both $\gamma$ and $\sigma$ are constant, (ii) an acceleration phase, in which $u_{\perp} \sim 1$ and the inertia of the charge carriers causes $\gamma$ to increase and $\sigma$ to decrease at the expense of the oscillating component of the field, and (iii) a coasting phase, in which the wave again proceeds at constant $\gamma$, which begins either when the current sheets 


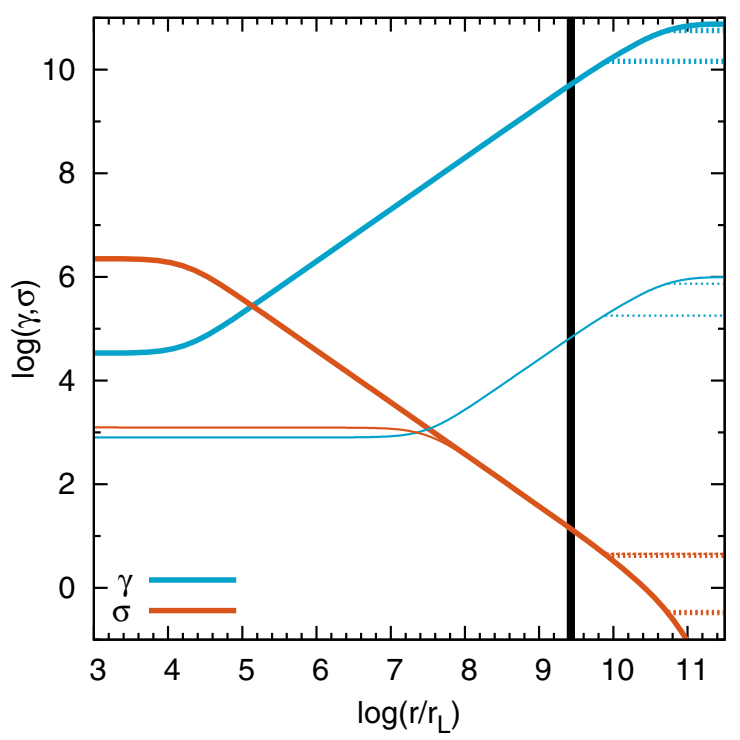

FIG. 1. The radial evolution of the magnetization parameter $\sigma$ and fluid Lorentz factor $\gamma$ in a pulsar wind for high pair loading ( $\mu=10^{6}$, thin lines) and low pair loading ( $\mu=a_{L}$, thick lines), for parameters corresponding to the $\operatorname{Crab}\left(a_{L}=7.6 \times 10^{10}\right)$. The position of the termination shock [26] is shown as a thick vertical line. For phase-averaged, dc magnetic fields equal to $90 \%$ and $50 \%$ of the field magnitude at launch, the horizontal, dotted lines show the solutions after the dissipation of the wave energy, i.e., in the regions $r \gtrsim 10^{10} r_{L}$ and $r \gtrsim 10^{11} r_{L}$, respectively.

merge (for a finite dc component) or is approached asymptotically as $\sigma \rightarrow 0$.

An approximate solution for the acceleration phase is [18]

$$
u_{\perp} \approx 1, \quad \gamma \approx 2 \mu r /\left(a_{L} r_{L}\right), \quad \sigma \approx r_{L} a_{L} /(2 r),
$$

valid for $a_{L} \gamma_{L} / \mu \ll r / r_{L} \ll a_{L}$, where $\gamma_{L}$ is the initial Lorentz factor of the wind. According to (3), inductive acceleration is a relatively slow process requiring an undisturbed pulsar wind that extends to a very large radius. For the Crab, Fig. 1 shows that $\sigma \approx 10$ at the termination shock, independent of $\mu$. Thus, only $10 \%$ of the Poynting flux is converted into kinetic-energy flux before the wind reaches the inner boundary of the nebula.

Taking into account that $B \approx(2 \pi m c / e P)\left(a_{L} r_{L} / r\right)$ for a magnetically dominated flow and that the termination shock compresses it by roughly a factor of 3 , the synchrotron emission of an electron that enters the nebula with $\gamma$ given by Eq. (3) peaks at a photon energy of

$$
h \nu_{\max } \approx 18 \mu^{2}(h / P)\left[r_{\mathrm{ts}} /\left(a_{L} r_{L}\right)\right] .
$$

Thus, for the Crab under average conditions $(P=33 \mathrm{~ms}$, $\left.r_{\text {ts }}=4.3 \times 10^{17} \mathrm{~cm}, \mu=10^{6}\right)$, particles that cross the shock initially radiate at $h \nu_{\max } \approx 10^{-1} \mathrm{eV}$ and are subsequently accelerated, either close to the shock or elsewhere in the nebula, to produce the time-averaged optical to hard x-ray synchrotron emission. However, the situation changes dramatically if the supply of particles in some section of the wind is interrupted. The arrival at the termination shock of a low-density pocket with $\mu=a_{L}$, as depicted in Fig. 1, causes the injection into the nebula of a radially directed beam that initially radiates photons of energy $h \nu_{\max } \approx 500 \mathrm{MeV}$.

An electron injected at pitch angle $90^{\circ}$ into a homogeneous magnetic field with a Lorentz factor such that its synchrotron peak is $h \nu_{\max }$ is deflected through an angle

$$
\delta \theta(\nu) \approx(80 \mathrm{MeV} / h \nu)\left(1-\nu / \nu_{\max }\right) \mathrm{rad}
$$

while cooling to the point at which its peak emission has decreased to $h \nu$. Thus, if energetic electrons are injected radially by a pulsar wind into the surrounding nebula, synchrotron photons of energy $h \nu>80 \mathrm{MeV}$ will appear to an observer with a sufficient angular resolution to emerge from a finite-sized patch on the termination shock, centered on the pulsar. The precise size and shape of this patch depend on the configuration of the magnetic field downstream of the termination shock, which is expected to be turbulent on length scales of $r_{\mathrm{ts}}$ [27] but, nevertheless, predominantly toroidal. A rough upper limit on the area of the patch, $A \lesssim \delta \theta^{2}(\nu) r_{\mathrm{ts}}^{2}$, follows from assuming random deflections through an angle of at most that given in Eq. (5). For $\nu \ll \nu_{\max }$, this implies $A \propto \nu^{-2}$. However, a weaker dependence on the frequency is found if the beam diverges diffusively.

This has important implications for the spectrum, time dependence, and overall power of the emission: Assume the pulsar wind is depleted of charges in a cone that occupies a solid angle $\Omega$ and includes the line of sight to the observer. Then, photons with energy $h \nu>h \nu_{t} \approx\left(80 / \Omega^{1 / 2}\right) \mathrm{MeV}$, that are radiated by electrons crossing the termination shock in this cone, remain within it, and their steady-state, differential flux has the form typical of monoenergetically injected, cooling electrons. On the other hand, lower-frequency photons emerge from a patch with $A>\Omega r_{\text {ts }}^{2}$ and are radiated into a solid angle that exceeds $\Omega$. This depletes the observed flux, leading to a turnover at $\nu_{t}$. Using (5) to estimate the rate at which the beam of cooling electrons diverges and employing a crude, monochromatic approximation for the synchrotron emissivity gives, for the differential energy flux $F_{\nu}$,

$$
\nu F_{\nu}= \begin{cases}f L_{\mathrm{sd}} /\left(8 \pi \sigma D^{2}\right)\left(\nu / \nu_{\max }\right)^{1 / 2} & \text { for } \nu_{t}<\nu<\nu_{\max }, \\ f L_{\mathrm{sd}} /\left(8 \pi \sigma D^{2}\right)\left(\nu_{t} / \nu_{\max }\right)^{1 / 2}\left(\nu / \nu_{t}\right)^{5 / 2} & \text { for } \nu<\nu_{t}\end{cases}
$$




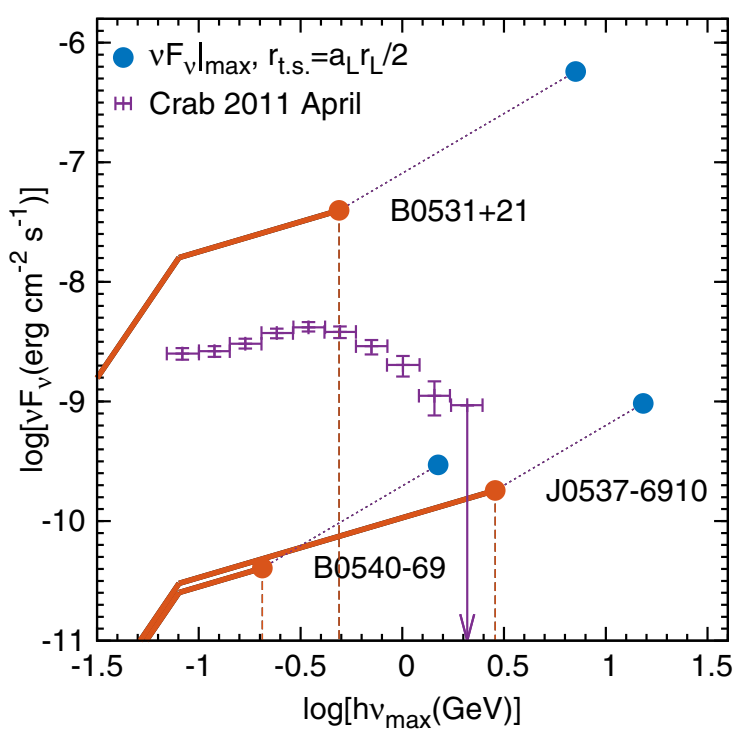

FIG. 2. The predicted flare spectrum [Eq. (6), solid lines], for the three most powerful known pulsars: the Crab $(\mathrm{B} 0531+21)$ and two objects in the Large Magellanic Cloud, assuming a turnover at $h \nu_{t}=80 \mathrm{MeV}$ and a filling factor $f=1$. Dotted lines trace the locus of the peak flux as the position of the termination shock is varied between the observed value (orange dots) and $a_{L} r_{L} / 2$ [blue dots (circles in black and white)]. Fermi observations of the powerful flare from the Crab Nebula in April 2011 are also shown-points taken from Fig. 6, epoch 7 of Ref. [3].

where $D$ is the distance to the source and the filling factor $f$ $(\leq 1)$ is the fraction of the flow containing charge-depleted regions. As shown in Fig. 2, observations of powerful gamma-ray flares from the Crab Nebula show a spectral form roughly consistent with (6), provided $h \nu_{\max }$ given by Eq. (4) is $500 \mathrm{MeV}$ and $\Omega$ is about 1 sr. Furthermore, the available power is more than adequate to explain the most powerful flare observed to date (that of April 2011 [3]): Within the uncertainties in the distance to this object and the angular distribution of power in the pulsar wind, this flare is consistent with $f \approx 0.1$ and $\sigma \approx 10$ at the termination shock, as indicated in Fig. 1. Gamma-ray flares from the $\mathrm{Crab}$ are seen with a range of powers and peak photon energies, and collations of their spectra, as presented, for example, in Figs. 6 and 7 of Ref. [3], can, in principle, be been used to test model predictions. However, although the crude model of synchrotron radiation plotted in Fig. 2 suggests rough agreement with the data, a more sophisticated approach would be needed to fit details of the spectrum.

Since electron-positron pairs are injected into the wind relatively close to the pulsar, their injection rate can, in principle, vary on time scales down to the rotation period or even shorter. A reduction in the injection rate on such time scales causes the wind to transition to a higher Lorentz factor. At the termination shock, the interface between the two flow states is broadened in time to roughly $r_{\text {ts }} / c \gamma^{2}$, where $\gamma$ refers to the slower flow, but, for fluctuations at the rotation rate, this is still very short $(\sim 10 \mathrm{~s}$ for the Crab) compared to the variation time of the flares. Therefore, both the leading edge and the trailing edge of a cone of chargedepleted wind can be treated as sharp transitions, and the observed rise and decay times of the emission are dominated by the variation in travel time from different parts of the illuminated patch on the termination shock. This patch appears larger at a lower photon energy, and the time scale $t_{\mathrm{var}}$ of variation is, correspondingly, energy dependent:

$$
\begin{aligned}
t_{\mathrm{var}} & =\delta \theta^{2}(\nu) r_{\mathrm{ts}} / c \\
& \approx 167(80 \mathrm{MeV} / h \nu)^{2}\left(1-\nu / \nu_{\max }\right)^{2} \text { days },
\end{aligned}
$$

where, in the second equation, the value of $r_{\text {ts }}$ for the Crab has been inserted. Thus, the time scale of $8 \mathrm{~h}$ observed in the powerful 2011 April Crab flare [3] suggests a patch of angular extent $\delta \theta \approx 2.5^{\circ}$. This is consistent with $h \nu \approx 400 \mathrm{MeV}$, provided $h \nu_{\max } \approx 500 \mathrm{MeV}$, which is also suggested by the spectrum shown in Fig. 2.

Although it provides an attractive scenario, the main shortcoming of the theory presented above is that it does not explain why the supply of charged particles to small parts of a magnetically dominated, relativistic outflow should suffer interruptions. The reason is assumed to lie in the physics of the electromagnetic cascades that are responsible for producing the pairs we observe when the flows terminate. These are known to be nonstationary, both in pulsars and in black hole magnetospheres [6,28], but a detailed understanding of their 3D spatial and temporal properties is not currently within reach. On the other hand, as long as the theory provides a reasonable fit to the data, it can be used to infer the properties of these cascades. Indeed, the fact that flares from the Crab can last for several days or longer implies that, during this time, pockets of depleted charge in a small cone of outflow directed at the observer have a spatial or temporal filling factor of several percent, which, in turn, suggests that the global geometry of the cascade varies on a time scale much longer than the pulsar rotation period.

The Crab is the most powerful pulsar known in the Milky Way, but it is not unique. In our neighboring galaxy, the Large Magellanic Cloud, two pulsars of comparable power are known: PSR J0537-6910 and PSR B0540-69 [29]. The predicted flare spectra for these objects are shown in Fig. 2, using estimates of the location of the termination shock given in Table 2 of Ref. [30]. Flares from the gammaray pulsar B0540-69 are not expected at energies significantly larger than $200 \mathrm{MeV}$ and may, therefore, be difficult to detect. PSR J0537-6910, however, has a shorter pulse period and a longer undisturbed wind than the Crab. As a result, its nebula (PWN N 157B) should exhibit synchrotron flares with photons of up to $3 \mathrm{GeV}$, as suggested by a recent analysis of Fermi data [31]. Thus, inductive spikes may be a common property of young, powerful pulsars and enable more detailed modeling and a deeper understanding 
of the way in which they energize their surrounding nebulae. Furthermore, inasmuch as they are also powered by low-density, magnetically dominated, relativistic outflows, both blazars and gamma-ray bursts may exhibit analogous phenomena [18].

This research was supported by a grant from the German-Israeli Foundation for Scientific Research and Development.

*john.kirk@mpi-hd.mpg.de gwenael.giacinti@mpi-hd.mpg.de

[1] M. Tavani et al., Science 331, 736 (2011).

[2] A. A. Abdo et al. (Fermi-LAT Collaboration), Science 331, 739 (2011).

[3] R. Buehler et al., Astrophys. J. 749, 26 (2012).

[4] P. W. Guilbert, A. C. Fabian, and M. J. Rees, Mon. Not. R. Astron. Soc. 205, 593 (1983).

[5] R. Bühler and R. Blandford, Rep. Prog. Phys. 77, 066901 (2014).

[6] B. Cerutti and A. M. Beloborodov, Space Sci. Rev. 207, 111 (2017).

[7] T. H. Hankins, J. A. Eilek, and G. Jones, Astrophys. J. 833, 47 (2016).

[8] V. Tatischeff et al., Proc. SPIE Int. Soc. Opt. Eng. 9905, 99052N (2016).

[9] M. J. Rees and J. E. Gunn, Mon. Not. R. Astron. Soc. 167, 1 (1974).

[10] J. G. Kirk, Y. Lyubarsky, and J. Petri, in Astrophysics and Space Science Library, edited by W. Becker (Springer, Berlin, Heidelberg, 2009), Vol. 357, p. 421.

[11] V. V. Usov, Astrophys. Space Sci. 32, 375 (1975).

[12] I. Arka and J. G. Kirk, Astrophys. J. 745, 108 (2012).

[13] F. V. Coroniti, Astrophys. J. 349, 538 (1990).

[14] Y. Lyubarsky and J. G. Kirk, Astrophys. J. 547, 437 (2001).

[15] J. G. Kirk and O. Skjæraasen, Astrophys. J. 591, 366 (2003).

[16] J. Zrake, Astrophys. J. 823, 39 (2016).
[17] H. C. Spruit and G. D. Drenkhahn, in Gamma-Ray Bursts in the Afterglow Era, Astronomical Society of the Pacific Conference Series Vol. 312, edited by M. Feroci, F. Frontera, N. Masetti, and L. Piro (Astronomical Society of the Pacific, San Francisco, 2004), p. 537.

[18] J. G. Kirk and I. Mochol, Astrophys. J. 729, 104 (2011); 736, 165(E) (2011).

[19] See Supplemental Material at http://link.aps.org/ supplemental/10.1103/PhysRevLett.119.211101 for a derivation of the radial evolution equations.

[20] O. Porth, R. Buehler, B. Olmi, S. Komissarov, A. Lamberts, E. Amato, Y. Yuan, and A. Rudy, Space Sci. Rev. 207, 137 (2017).

[21] B. Olmi, L. Del Zanna, E. Amato, and N. Bucciantini, Mon. Not. R. Astron. Soc. 449, 3149 (2015).

[22] N. Bucciantini, J. Arons, and E. Amato, Mon. Not. R. Astron. Soc. 410, 381 (2011).

[23] A. Tchekhovskoy, A. Philippov, and A. Spitkovsky, Mon. Not. R. Astron. Soc. 457, 3384 (2016).

[24] R. Buckley, Mon. Not. R. Astron. Soc. 180, 125 (1977).

[25] I. Contopoulos and D. Kazanas, Astrophys. J. 566, 336 (2002).

[26] J. J. Hester, K. Mori, D. Burrows, J. S. Gallagher, J. R. Graham, M. Halverson, A. Kader, F. C. Michel, and P. Scowen, Astrophys. J. 577, L49 (2002).

[27] O. Porth, M. J. Vorster, M. Lyutikov, and N. E. Engelbrecht, Mon. Not. R. Astron. Soc. 460, 4135 (2016).

[28] A. Levinson and N. Segev, arXiv:1709.09397.

[29] M. Ackermann et al. (Fermi-LAT Collaboration), Science 350, 801 (2015).

[30] O. Kargaltsev and G. G. Pavlov, in 40 Years of Pulsars: Millisecond Pulsars, Magnetars and More, American Institute of Physics Conference Series Vol. 983, edited by C. Bassa, Z. Wang, A. Cumming, and V. M. Kaspi (AIP, New York, 2008), pp. 171-185.

[31] S. Saito, D. Khangulyan, K. Hagiwara, and Y. Uchiyama, in Proceedings of the 6th International Symposium on High Energy Gamma-Ray Astronomy, American Institute of Physics Conference Series Vol. 1792 (AIP, New York, 2017), p. 040013. 